\section{DIN EN ISO 15194}

G. Schumann

Hannover, Deutschland

\section{Englischer Begriff ISO 15194}

Beschreibung Die internationale Norm „In-vitro-Diagnostika - Messung von Größen in Proben biologischen Ursprungs - Anforderungen an zertifizierte Referenzmaterialien und an den Inhalt der Begleitdokumentation" enthält die Anforderungen an die Beschreibung von Referenzmaterial. Zertifiziertes Referenzmaterial dient als Kalibriermaterial für
Referenzmessverfahren oder als Material zu deren Kontrolle. Die Zertifizierung von primärem Referenzmaterial ist metrologischen Instituten vorbehalten. Spezielle Messverfahren zur Charakterisierung der primären Referenzmaterialien haben größte Genauigkeit und kleinste Messunsicherheit.

\section{Literatur}

DIN EN ISO 15194 (2009) In-vitro-Diagnostika - Messung von Größen in Proben biologischen Ursprungs - Anforderungen an zertifizierte Referenzmaterialien und an den Inhalt der Begleitdokumentation. Beuth-Verlag, Berlin 